Pinney mention potential bias. We believe that the most important potential bias in this study, as indicated in the discussion section, could have arisen from the selection of the referent population. This bias was minimised by using a matched pair design. A randomly sampled referent group would tend to reflect the characteristics of the underlying referent population, whereas the referent group chosen by matching ends up being more like the index group than like the underlying population of referents being sampled. In particular, matching generally precludes the evaluation of the underlying population relations between the matching variables and exposure state in a follow up study.'

With respect to the selection of the exposed population, they were composed of volunteers, many of whom had received or were asking for compensation for work related disorders. This subgroup of the total population was certainly not representative of the entire workforce between 1971 and 1984 and no pretentions were made to this effect or to the effect that they were representative of the entire population of women workers in the microelectronic industry. The study considered the question: Was there evidence of adverse reproductive outcomes among this group of former women workers?

Upfal and Pinney state that "no attempts to validate the outcomes were cited for even a sample of exposed workers and referents." In the discussion, we indicate that adverse reproductive outcomes among the former workers were verified through medical records. It seems obvious that workers who are concerned in litigation as plaintiffs could have a strong interest in the recognition and documentation of possible work related health problems and that is precisely why we paid attention to this validation. Although there were minor inconsistencies in reported miscarriages, there were no errors in the number of women having had at least one miscarriage before or after employment. If former women workers had minimised their spontaneous abortions before employment, we should have found during this period a consistent inbalance among the discordant pairs. This is not the case as we found $10 v 11$ discordant pairs.

Upfal and Pinney point out that no attempt was made to match on number of prior fetal losses. We agree that it would have been convenient to match on prior fetal losses. On the other hand, if we had done this, we would not have been able to verify the above as no discordant pairs would have occurred. As stated in our article, however, "To eliminate the potential effect of overestimation of workers who are at increased risk to have spontaneous abortion in one of the two groups we excluded pairs for which one of the members suffered at least one spontaneous abortion before employment (table 3 ). The odds ratio decreased somewhat $(\mathrm{OR}=4)$ but remained significant $\left(\chi^{2}=5 \cdot 4 ; \mathrm{p}<\right.$ $5 \%$ ).",

Finally, the authors of the letter mention twice that the study is based on 25 pregnancies during employment, which is a misunderstanding as it is 25 pairs of mothers who contributed to the study, a total of $2 \times 63$ pregnancies (the number of pregnancies is equal by chance!). Moreover, the OR was not estimated from these 25 pairs in which each member had had at least one pregnancy during the period covering the former worker's employment, but from the 33 pairs in which each member had had at least one pregnancy after the start of employment. These 33 pairs contributed to a total of 81 (of which 32 terminated in miscarriages) and 77 pregnancies (of which five terminated in miscarriages) in the former microelectronic and referent groups respectively.

We mention in the article that when one considers strictly the 25 pairs of child bearing women during the period of employment, the OR (15) was highly significant. We also indicate that this OR had a weak precision to minimise possible hasty interpretation. The constant repetition in Upfal's and Pinney's letter of "meagre" or "small" sample size suggests to the reader that there is a lack of power of the tests used. This is refuted by the fact that significance levels are consistently surpassed.

In conclusion, we believe that we have been very careful about the interpretation given to these findings and particularly about the causal factor suspected (multiple organic solvent exposure). Although this is far from being a definitive study on reproductive outcomes in the microelectronic industry, it does indicate evidence of adverse reproductive outcomes among this group of former microelectronic workers, possibly related to their employment. In the discussion we stated that "this increased frequency of miscarriages seems to be associated with employment in this microelectronic assembly plant." We believe strongly that more studies are needed among active and past women workers in this worldwide industry, where working conditions and exposures differ greatly from country to country.

1 Kleinbaum DG, Kupper LL, Morgenstern H. Epidemiologic research: principles and quantitative methods. Belmont CA: Lifetime Learning Publications, 1982:377-402.

\section{Exercise capacity in coal workers' pneumoconiosis: an analysis using causal modelling}

Sir,-We were interested in the paper by Cooper and Johnson (1990;47: 52-7) using causal modelling to identify factors that might be used to assess disability in coal workers. Their paper reflected the 'Alice through the looking glass' situation in the United States where disability is defined by politicians and lawyers without much regard to reality. Hence their statement (Correspondence 1990; 47:574-5) that disability is a legal not a medical term and cannot be defined. This may be true of handicap. Respiratory disability has, however, been defined by the World Health Organisation as a reduction in exercise capacity due to impaired lung function. 'As with other physiological tests the reduction can be assessed in terms of the reference values for exercise capacity in healthy men and it can be scored after the manner proposed by the American Thoracic Society (ATS) for indices of lung function. ${ }^{2}$ Using the ATS approach zero disability is when the exercise capacity exceeds the lower limit of normal and $100 \%$ disability can be defined as inability to increase the oxygen uptake above twice the resting level. This is the situation when the subject is unable to walk more than a few yards before being stopped by incapacitating breathlessness. A linear rating scale for respiratory disability with the above limits has been proposed by a working group of the European Society for Clinical Respiratory Physiology. The scores so obtained have been found to agree well with the disability ratings awarded by a DHSS Medical Board- 
ing Centre for occupationally related respiratory diseases. ${ }^{3}$ This approach to disability assessment is within the medical domain and capable of objective evaluation. United States legislators should consider a similar approach.

\section{JE COTES} B KING

University of Newcastle upon Tyne, Division of Environmental and Occupational Medicine, Respiration and Exercise Laboratory, Ridley Building, The University, Newcastle upon Tyne, NE1 7RU

1 World Health Organisation. International classification of impairments, disabilities and handicaps. Geneva: WHO, 1980

2 American Thoracic Society. Evaluation of impairment/disability secondary to respiratory disease. Am Rev Respir Dis 1982;126:945-51.

3 Cotes JE. Rating respiratory disability: a report on behalf of a working group of the European Society of Clinical Respiratory Physiology. Eur Respir J 1990;3:1074-7.

\section{NOTICES}

A seminar on welding fume, the Palais de Congrès, Strasbourg, France, 8-9 April 1991.

The event is the first joint venture between The Welding Institute, United Kingdom and its French counterpart, the Institut de Soudure. The seminar is a unique opportunity for everyone concerned with welding and its safety to benefit from the knowledge and experience of world experts on: welding fume-the current situation, the health effects of fume, characterisation and assessment of fume exposure, control of fume hazards in the workplace.

Delegates will gain a greater understanding of welding fume and learn how it can be controlled for improved safety. Presentations will be given by invited speakers from France, Germany, Italy, United Kingdom, and Soviet Russia. A French German English simultaneous translation will be transmitted throughout the seminar. For further information please contact The Meetings Department, The Welding Institute, Abington Hall, Abington, Cambridge CB1 6AL. Tel: 0223891162. 22nd French National Meeting and 1st European Meeting of Occupational Medicine, Nantes, France, 2-5 June 1992

Programme: (1) Occupational medicine and the environment; (2) Health, ageing, and work; (3) Occupational dermatosis of chemical origin.

The organising committee consists of The Society of Occupational Medicine and Ergonomics of the West, The Occupational Medicine Institute of Nantes, and The Occupational Medicine Institute of Rennes. The official language will be French with simultaneous translation into English. For details contact the Secretary General: Dr Catherine Butat, Société de Médecine du Travail et d'Ergonmie de l'Ouest, Faculté de Médecine - 1, rue Gaston Veil, 44035 Nantes Cedex, France.

Industrial audiometry, Wendover Hotel, Monton Road, Eccles, Manchester, 10-12 April, 15-17 May 1991.

These three day courses offer training in audiometry for industrial medical staff, safety officers, and others concerned with hearing in industry. The courses cover the theory and practice of audiometry, the general background to hearing conversation, and provide practical tuition on a range of modern audiometers. Details from Dr W ' Tempest, Kismet, Croyde Road, St Annes, Lancs FY8 1EX. 